\title{
Mudança climática e seus efeitos na cultura do arroz
}

\author{
Climate change and its effects on rice
}

\author{
Lidiane Cristine Walter ${ }^{\mathrm{I}}$ Nereu Augusto Streck ${ }^{\mathrm{II}}$ Hamilton Telles Rosa ${ }^{\mathrm{III}}$ \\ Cleusa Adriane Menegassi Bianchi Krüger ${ }^{\mathrm{III}}$
}

\section{- REVISÃO BIBLIOGRÁFICA -}

\section{RESUMO}

\begin{abstract}
A partir da revolução industrial, houve um aumento da emissão de gases de efeito estufa na atmosfera terrestre, como o dióxido de carbono ( $\mathrm{CO}_{2}$ ), o que poderá levar a um aumento na temperatura global até o final do século XXI. O efeito direto do incremento na concentração de $\mathrm{CO}_{2}$ nas plantas é a possibilidade de aumento da taxa de crescimento das plantas e produtividade das culturas, uma vez que o $\mathrm{CO}_{2}$ é o substrato para fotossintese. Se o aumento da concentração de $\mathrm{CO}_{2}$ for acompanhado de aumento da temperatura do ar, poderá haver encurtamento do ciclo e aumento da respiração do tecido vegetal, reduzindo ou anulando os efeitos benéficos do $\mathrm{CO}_{2}$. No entanto, a resposta aos aumentos na concentração de $\mathrm{CO}_{2}$ e temperatura do ar varia de acordo com a cultura considerada. Assim, o objetivo desta revisão foi reunir informações da resposta ecofisiológica da cultura do arroz, um dos três cereais mais produzidos e consumidos pela população mundial, à mudança climática. Plantas com metabolismo $C_{3}$, como o arroz, são mais beneficiadas pelo aumento da concentração de $\mathrm{CO}_{2}$ atmosférico do que plantas com metabolismo $C_{4}$. Altas temperaturas diurnas e noturnas podem reduzir drasticamente o potencial produtivo da cultura do arroz devido ao encurtamento do ciclo da cultura e à esterilidade de espiguetas. Essa tendência pode ser mitigada com a seleção de genótipos mais resistentes às condições de alta temperatura do ar durante oflorescimento, bem como a alteração da época de semeadura.
\end{abstract}

Palavras-chave: Oryza sativa, aquecimento global, ecofisiologia, produção de alimentos, estresse.

\section{ABSTRACT}

Since the industrial revolution there was an increase in the atmospheric greenhouse gases, such as the carbon dioxide
$\left(\mathrm{CO}_{2}\right)$, which may lead to an increase in global temperature by the end of the $21^{\text {th }}$ century. The direct effect of the increase in the $\mathrm{CO}_{2}$ concentration on plants is the possibility of increasing plant growth rate and crop yield, once $\mathrm{CO}_{2}$ is the substrate for photosynthesis. If the increase in $\mathrm{CO}_{2}$ concentration will be accompanied by an increase in air temperature, there may not be an increase in plant growth and crop yield because of a shortening of the developmental cycle an increase in plant tissue respiration. However, the response to elevated $\mathrm{CO}_{2}$ and temperature varies depends upon the crop. Thus, the objective of this review is to assemble information about the ecophysiological response of rice crop, one of the tree major cereals produced and consumed by the world population, to climate change. Plants with the $C_{3}$ metabolism, as rice, are more benefitted from the increase in atmospheric $\mathrm{CO}_{2}$ concentration than plants with the $\mathrm{C}_{4}$ metabolism. High daytime and nighttime temperatures may drastically reduce the yield potential of rice crop due to shortening of the developmental cycle and spikelet sterility. Such a trend can be mitigated by selecting genotypes more resistant to conditions of high air temperature during flowering and by changing the sowing date.

Key words: Oryza sativa, global warming, ecophisiology, food production, stress.

\section{INTRODUÇÃO}

A partir da revolução industrial, em meados do século XVIII, a humanidade passou por um processo de transformação acompanhado por uma

'Programa de Pós-graduação em Engenharia Agrícola, Centro de Ciências Rurais (CCR), Universidade Federal de Santa Maria (UFSM), Santa Maria, RS, Brasil.

"Departamento de Fitotecnia, CCR, UFSM. Av. Roraima, 1000, 97105-900, Santa Maria, RS, Brasil. E-mail: nstreck1@smail.ufsm.br. Autor para correspondência.

II'Programa de Pós-graduação em Agronomia, CCR, UFSM, Santa Maria, RS, Brasil. 
grande evolução tecnológica, na qual ocorreu a mecanização dos sistemas de produção e uso crescente de combustíveis fósseis em diversos setores. Em razão dessa mudança, houve aumento da emissão de gases de efeito estufa, como dióxido de carbono $\left(\mathrm{CO}_{2}\right)$, metano $\left(\mathrm{CH}_{4}\right)$ e óxido nitroso $\left(\mathrm{N}_{2} \mathrm{O}\right)$, na atmosfera terrestre. Assim, as concentrações desses gases aumentaram em consequência das atividades antropogênicas desde 1750 , passando de valores pré industriais de $280 \mathrm{ppm}$ para 379ppm de $\mathrm{CO}_{2}, 715 \mathrm{ppb}$ para $1774 \mathrm{ppb}$ de $\mathrm{CH}_{4} \mathrm{e}$ 270 ppb para 319ppb de $\mathrm{N}_{2} \mathrm{O}$ em 2005 (IPCC, 2007).

$\mathrm{O}$ efeito estufa é um processo natural da atmosfera terrestre, no qual parte da radiação de ondas longas, emitida pela superfície terrestre, é absorvida pelos gases de efeito estufa da atmosfera e posteriormente é emitida também na forma de ondas longas, em direção à superfície terrestre. Esse processo é fundamental para a manutenção da temperatura e consequentemente da vida no Planeta. No entanto, o aumento da concentração desses gases pode intensificar o processo, desestabilizando o equilíbrio energético no planeta e originando o fenômeno conhecido como Aquecimento Global. Os principais gases de efeito estufa são o vapor d'água $\left(\mathrm{H}_{2} \mathrm{O}\right)$, o $\mathrm{CO}_{2},{\mathrm{o} \mathrm{CH}_{4}}_{4}$ o $\mathrm{N}_{2} \mathrm{O}$. Várias atividades humanas contribuem para o aumento da concentração de $\mathrm{CO}_{2}$, $\mathrm{CH}_{4}$ e $\mathrm{N}_{2} \mathrm{O}$ na atmosfera, entre elas destaca-se o uso de combustíveis fósseis (carvão, petróleo e gás natural), as queimadas e a agricultura.

Uma forma de medir a evolução da concentração dos gases da atmosfera terrestre nos últimos mil anos é por meio de análises das bolhas de ar, contidas em testemunho de gelo na Antártica, onde se destaca o forte aumento da concentração de $\mathrm{CO}_{2}$ no século XX (MANN et al., 1998; MANN et al., 1999). Atualmente, a taxa de aumento da concentração de $\mathrm{CO}_{2}$ é de 1,9ppmv por ano (IPCC, 2007). Com isso, espera-se que a atual concentração venha a duplicar em algum momento deste século, podendo acarretar vários efeitos sobre as atividades humanas, incluindo a agricultura (STRECK, 2005).

$\mathrm{O}$ efeito direto do incremento na concentração de $\mathrm{CO}_{2}$ na agricultura é a possibilidade de aumento da taxa de crescimento e produtividade das culturas no futuro, uma vez que o $\mathrm{CO}_{2}$ é o substrato primário para a fotossíntese (ROSENBERG et al., 1983; TAIZ \& ZEIGER, 2004; STRECK, 2005). No entanto, o aumento da concentração de $\mathrm{CO}_{2}$ pode causar uma elevação na temperatura do ar, que, segundo projeções do último relatório do IPCC, podem chegar a $6,4^{\circ} \mathrm{C}$ até o final do século XXI (IPCC, 2007). No Brasil, GONÇALVES \& ASSAD (2009), ao analisar séries históricas de diversas estações meteorológicas, encontraram um aumento significativo nas temperaturas mínimas do ar em todo o país, com maior impacto na região Norte, onde a tendência de aumento é de $0,5^{\circ} \mathrm{C}$ por década. Para a região Sul do Brasil, o acréscimo na temperatura mínima do ar foi de $0,2^{\circ} \mathrm{C}$ por década. Assim, se o aumento na concentração de $\mathrm{CO}_{2}$ na atmosfera vier acompanhado de aumento na temperatura do ar, os efeitos benéficos do $\mathrm{CO}_{2}$ poderão ser reduzidos ou anulados, com resposta variável para as diferentes espécies de plantas (SIQUEIRA et al., 2001; STRECK, 2005). A mudança climática, segundo AGGARWAL \& MALL (2002), ainda pode causar efeitos indiretos na produção das culturas, como alterações na disponibilidade de água para irrigação, competição com pragas, doenças e plantas invasoras, alterações na fertilidade do solo e erosão.

Devido à importância da mudança climática sobre as plantas, a comunidade científica tem se dedicado a estudar seus possíveis impactos no crescimento, desenvolvimento e produtividade das culturas. A maior concentração de produção e consumo de arroz no mundo encontra-se na Ásia (MATTHEWS et al., 1997) e, por este motivo, também é na Ásia que encontra-se a maior parte das pesquisas referentes à cultura do arroz, pois possíveis efeitos negativos da mudança climática sobre a cultura do arroz são preocupação constante dos pesquisadores asiáticos, em função da segurança alimentar.

O objetivo desta revisão foi reunir informações a respeito da resposta ecofísiológica da cultura do arroz à mudança climática.

\section{A cultura do arroz e a mudança climática}

O arroz (Oryza sativa L.) é um dos três cereais mais produzidos e consumidos no mundo, ficando atrás apenas do trigo e do milho (USDA, 2009). Faz parte da dieta básica de aproximadamente $50 \%$ da população mundial (LUZZARDI et al., 2005), podendo ser considerado o mais importante para a alimentação humana, pois, dentre essas três culturas, o arroz é a única consumida diretamente, enquanto o trigo e o milho são processados pela indústria, ou são utilizados na alimentação animal (SHEEHY et al., 2007).

Plantas com metabolismo $\mathrm{C}_{3}$, como o arroz, são mais beneficiadas pelo aumento da concentração de $\mathrm{CO}_{2}$ atmosférico do que plantas com metabolismo $\mathrm{C}_{4}$ (SIQUEIRA et al., 2001; STRECK, 2005), pois as últimas, devido a modificações morfofisiológicas no aparato fotossintético, já utilizam com eficiência o $\mathrm{CO}_{2}$ presente no ar atmosférico nas concentrações atuais. FLOSS (2008) cita que, em condições de campo, com boa luminosidade, a quantidade de $\mathrm{CO}_{2}$ atmosférico é em torno de $337 \mathrm{ppm}$, o que quase sempre limita a 
fotossíntese nas plantas com metabolismo $\mathrm{C}_{3}$. Este fato é agravado durante o período do dia em que a disponibilidade de radiação solar é maior, pois a planta realiza fotossíntese e consome rapidamente o $\mathrm{CO}_{2}, \mathrm{o}$ que determina uma diminuição no conteúdo de $\mathrm{CO}_{2}$ próximo às folhas.

Efeitos do aumento da concentração de $\mathrm{CO}_{2}$ na cultura do arroz

Como a atual concentração de $\mathrm{CO}_{2}$ atmosférico é limitante para a fotossíntese das plantas $\mathrm{C}_{3}$, a taxa fotossintética dessas plantas aumenta cerca de $66 \%$ com o dobro da atual concentração de $\mathrm{CO}_{2}$, respondendo positivamente aos incrementos até aproximadamente $1000 \mathrm{ppm}$ de $\mathrm{CO}_{2}$ (TAIZ \& ZEIGER, 2004). No entanto, o aumento na taxa fotossintética não significa um aumento equivalente no rendimento de grãos, pois parte da energia é utilizada no metabolismo da planta e no crescimento das demais partes dela, como colmos e folhas. A maioria das pesquisas indica um aumento de aproximadamente $30 \%$ no rendimento de espécies $\mathrm{C}_{3}$, inclusive para o arroz, em função do dobro da concentração de $\mathrm{CO}_{2}$ (KIMBALLet al., 1993; SOLTANI et al., 2001; STRECK, 2005). No entanto, em alguns estudos foram encontrados aumentos maiores no rendimento de grãos de arroz, como um incremento de mais de $56 \%$ relatado por KRISHNAN et al. (2007), em estudo de simulação com o modelo InfoCrop no leste da Índia, usando uma cultivar de ciclo precoce. Resultados experimentais mostram aumentos na biomassa seca e rendimento comercial de algumas culturas em resposta ao dobro da concentração do $\mathrm{CO}_{2}$, sendo o incremento médio de $23,32,42,54$ e $52 \%$ para frutas, cereais $\mathrm{C}_{3}$, folhosas, legumes e raízes, respectivamente (STRECK, 2005).

Em um estudo de três anos na China, sob condições de ambiente controlado, aplicando 200ppm de $\mathrm{CO}_{2}$ acima da concentração ambiente, YANG et al. (2006) encontraram um incremento na produção de matéria seca (MS) que levou a um aumento médio de $13 \%$ no rendimento de grãos. O incremento na MS variou com o estágio de desenvolvimento da cultura, com uma tendência de redução no incremento à medida que a cultura avança no seu desenvolvimento. Quanto à distribuição da $\mathrm{MS}$, com o aumento do $\mathrm{CO}_{2}$, houve uma redução significativa na fração da MS das folhas e aumento na fração da MS dos colmos. $\mathrm{O}$ incremento final da biomassa foi maior do que o incremento no rendimento de grãos.

Efeitos do aumento da temperatura do ar na cultura do arroz

Se o aumento da concentração de $\mathrm{CO}_{2}$ for acompanhado de aumento da temperatura do ar, poderá não haver aumento no crescimento e produtividade das culturas, devido ao encurtamento do ciclo (WIEGAND \& CUELLAR, 1981; SIONIT et al., 1987; SIQUEIRA et al., 2001) e aumento da respiração (ROSENBERG et al., 1983; LONG, 1991; VU et al., 1997; TAIZ \& ZEIGER, 2004), ou seja, as plantas consumiriam mais energia na respiração, reduzindo os fotoassimilados disponíveis para os órgãos de reserva da planta. No Brasil, estudos indicam um aumento das áreas de risco climático para a cultura do arroz irrigado, devido ao aumento da temperatura do ar (ASSAD \& PINTO, 2008).

$\mathrm{Na}$ China, TAO et al. (2006) identificaram uma elevação da temperatura, medida entre 1981-2000, que afetou a fenologia e o rendimento de grãos das culturas. Nesse caso, houve redução da produtividade com o aumento da temperatura máxima, pela maior esterilidade de espiguetas no arroz, e com o aumento da temperatura mínima, pelo aumento da taxa de respiração noturna e, consequentemente, consumo de biomassa.

Para as condições do noroeste da Índia, LAL et al. (1998) avaliaram a vulnerabilidade da cultura do arroz à futura mudança no clima, utilizando o modelo CERES. Nessa região, está previsto que, até a metade do século XXI, o aumento na concentração de $\mathrm{CO}_{2}$ atmosférico, de 330 para $660 \mathrm{ppm}$, cause um aumento de 1 a $2^{\circ} \mathrm{C}$ na temperatura média diária do ar, além de redução de $0,5 \mathrm{~mm} \mathrm{dia}^{-1}$ na precipitação. Os efeitos positivos do dobro da concentração de $\mathrm{CO}_{2}$ no rendimento do arroz seriam anulados com o aumento de apenas $2^{\circ} \mathrm{C}$ na temperatura do ar.

Ainda na Índia, os efeitos da mudança climática na cultura do arroz foram estudados por AGGARWAL \& MALL (2002) através de modelos matemáticos, considerando incrementos graduais de $\mathrm{CO}_{2}$ e temperatura, sob diferentes doses de nitrogênio. Similarmente aos demais trabalhos, esses autores encontraram aumento no rendimento do arroz com maior concentração de $\mathrm{CO}_{2}$ na atmosfera, mas este efeito positivo foi anulado quando o aumento da temperatura do ar foi considerado no estudo. Na tabela 1, estão os valores do incremento de temperatura do ar que são necessários para anular os efeitos positivos do $\mathrm{CO}_{2}$ sobre a produtividade do arroz, em diferentes locais da Índia (AGGARWAL \& MALL, 2002). Em regiões onde as doses de nitrogênio utilizadas são insuficientes para suprir as necessidades da cultura, a maior concentração de $\mathrm{CO}_{2}$ na atmosfera não terá um grande efeito no rendimento de grãos de arroz. Isso ocorre porque há menor fotossíntese devido à menor quantidade de $\mathrm{N}$, que é o principal constituinte dos cloroplastos (TAIZ \& ZEIGER, 2004). 
Tabela 1 - Aumento na temperatura $\left({ }^{\circ} \mathrm{C}\right)$ que anula os efeitos positivos da concentração de $\mathrm{CO}_{2}$ em diferentes regiões e dois níveis de manejo. Adaptado de AGGARWAL \& MALL (2002).

\begin{tabular}{llccc}
\hline \multirow{2}{*}{ Local } & \multicolumn{1}{c}{$\begin{array}{c}\text { Nível } \\
\text { de Manejo* }\end{array}$} & \multicolumn{3}{c}{----Concentração de $\mathrm{CO}_{2}$---- } \\
& $450 \mathrm{ppm}$ & $550 \mathrm{ppm}$ & $650 \mathrm{ppm}$ \\
\hline Norte da Índia & Melhorado & 1,7 & 3,2 & $>5,0$ \\
& Atual & 1,9 & 2,7 & 4,8 \\
Leste da Índia & & & & \\
& Melhorado & 1,2 & 1,5 & $>5,0$ \\
& Atual & 2,0 & 4,4 & $>5,0$ \\
Oeste da Índia & Melhorado & 0,9 & 1,8 & 2,8 \\
& Atual & 1,0 & 2,1 & 3,4 \\
& & & & \\
Sul da Índia & Melhorado & 1,0 & 2,3 & 4,4 \\
& Atual & 0,9 & 2,0 & 3,4 \\
\hline
\end{tabular}

*O nível de manejo Atual refere-se à aplicação de $150 \mathrm{~kg} \mathrm{~N} \mathrm{ha}^{-1}$ em três doses e irrigações frequentes, uma prática comum em arroz irrigado na Índia. O nível Melhorado simula um manejo de produção potencial daqui a algumas décadas, considerando o provável desenvolvimento econômico e tecnológico da agricultura.

Na tabela 1, pode-se observar ainda que o aumento de temperatura, para anular os efeitos positivos do aumento de $\mathrm{CO}_{2}$, varia com a concentração de $\mathrm{CO}_{2}$ atmosférico de acordo com o local e com o nível de manejo da lavoura arrozeira, variando de $0,9^{\circ} \mathrm{C}$ no Oeste e Sul da Índia, com concentração de $\mathrm{CO}_{2}$ de $450 \mathrm{ppm}, \mathrm{a}>5^{\circ} \mathrm{C}$ no Leste e Norte da Índia, com concentração de $\mathrm{CO}_{2}$ de $650 \mathrm{ppm}$. Chama a atenção, nos dados da tabela 1, o fato do "Manejo Melhorado", que representa uma possível mudança tecnológica na cadeia produtiva do arroz na Índia, alterar o aumento de temperatura que anula os efeitos benéficos do aumento do $\mathrm{CO}_{2}$ de forma diferente, dependendo da região de cultivo, ora sendo maior do que o nível de manejo atual (Sul da Índia), ora sendo menor (Norte, Leste e Oeste da Índia). Esses resultados reforçam que há interação entre vários fatores que afetam a produtividade das culturas agrícolas, no caso deste trabalho, a cultura do arroz, indicando alta vulnerabilidade dos ecossistemas agrícolas à mudança climática, em que soluções e práticas de mitigação devem ser consideradas sítio-específicas.

Efeitos da mudança climática na fase vegetativa do arroz

O ciclo de desenvolvimento da cultura do arroz pode ser dividido em duas fases: vegetativa e reprodutiva (STRECK et al., 2006), em que a fase vegetativa é compreendida entre a emergência e o aparecimento da última folha (folha bandeira) no colmo principal da planta de arroz, ou seja, corresponde ao período de emissão de folhas da cultura. A duração dessa fase é a principal responsável pela duração do ciclo total da cultura e é afetada principalmente pela temperatura do ar (STRECK et al., 2006). Assim, o aumento na temperatura tem um potencial de afetar significativamente o ciclo de desenvolvimento da cultura, enquanto o aumento da concentração de $\mathrm{CO}_{2}$ atmosférico poderá aumentar a taxa fotossintética.

Em cenários de mudança climática para a China, TAO et al. (2008) reportam $100 \%$ de probabilidade de diminuição do ciclo da cultura com aumento de temperatura, diminuições essas tanto maiores quanto maior o aumento da temperatura média do ar. Estes resultados concordam com os relatados por LAGO et al. (2008) no Brasil, para diferentes fases de desenvolvimento de diferentes cultivares, em cenários com aumentos simétrico e assimétrico na temperatura do $\operatorname{ar}\left(\right.$ de 1 a $\left.5^{\circ} \mathrm{C}\right)$, utilizando um modelo não linear para simular o desenvolvimento da cultura. Os últimos autores reportam uma diminuição da fase vegetativa (emergência - diferenciação do primórdio floral) nos cenários com aumentos de temperatura simétricos (aumentos iguais na temperatura mínima e máxima do ar) e assimétricos (temperatura mínima aumenta mais do que a máxima), com as cultivares de origem indica sendo mais sensíveis que os de origem japonica es biótipos de arroz vermelho.

A duração do ciclo total dos biótipos de arroz vermelho diminuiu em quase todos os cenários de mudança climática utilizados por LAGO et al. (2008), podendo ser um indício de aumento da competitividade dessa importante planta daninha da cultura do arroz no RS em cenários de mudança climática, mostrando maior plasticidade e capacidade de adaptação, em função de sua ampla base genética. ZISKA \& McCLUNG (2008), em experimentos em ambiente natural, identificaram uma vantagem competitiva de plantas de arroz vermelho sobre arroz cultivado, em função do maior crescimento inicial de folhas e raízes, maior biomassa total e área foliar no estágio de iniciação da panícula da invasora. Quando as plantas foram avaliadas em ambiente com 500ppm de $\mathrm{CO}_{2}$, o arroz vermelho apresentou vantagem competitiva ainda maior do que no ambiente natural, demonstrando sua maior plasticidade genética e fenotípica (ZISKA \& McCLUNG, 2008). Os resultados desses estudos de mudança climática com arroz vermelho indicam que esta invasora poderá se tornar ainda mais competitiva em cenários futuros, impondo uma maior limitação no rendimento de grãos do arroz cultivado, com o aumento da concentração de $\mathrm{CO}_{2}$ atmosférico. 
Em outro trabalho realizado no Brasil, STEINMETZ et al. (2006) estudaram o impacto da mudança climática sobre a duração da fase vegetativa do arroz irrigado para as principais regiões produtoras do Rio Grande do Sul. Os autores, utilizando o método de Graus-dia, com temperatura base de $11^{\circ} \mathrm{C}$, encontraram diminuições progressivas na duração da fase vegetativa com o aumento da temperatura do ar, à medida que a data de emergência avança (Tabela 2). Isso pode implicar diminuição do perfilhamento, diminuição do número de folhas e consequentemente menor produtividade das culturas.

Efeitos da mudança climática na fase reprodutiva da cultura do arroz

A segunda fase do ciclo de desenvolvimento da cultura do arroz é a fase reprodutiva, correspondendo ao período entre a emissão da última folha e a maturidade fisiológica (STRECK et al. 2006). Durante essa fase, dois importantes componentes do rendimento são definidos: número de grãos por panícula e peso de grãos. O número de grãos por panícula pode ser modificado pela esterilidade de espiguetas em função das temperaturas extremas, tanto muito baixas, quanto muito elevadas (MATSUI et al., 1997). O peso de grãos é dependente da quantidade de fotoassimilados que é translocada para o enchimento de grãos, estando, portanto, relacionado com a taxa fotossintética $\left(\mathrm{CO}_{2}\right)$ e com a duração desta fase (temperatura).

Em diferentes locais da Índia, KRISHNAN et al. (2007) simularam o rendimento de grãos de arroz em cenários de mudança climática, com aumentos na temperatura do ar de até $5^{\circ} \mathrm{C}$, e os resultados indicam que o rendimento de grãos de arroz diminuiu em relação ao cenário atual para locais tropicais de latitudes entre 20 e $23^{\circ} \mathrm{N}$, enquanto o rendimento de grãos nos cenários de mudança climática aumentou em um local extratropical com latitude de $26^{\circ} 8^{\prime} \mathrm{N}$. Resultados similares já haviam sido relatados anteriormente por SOLTANI et al. (2001), sugerindo que o rendimento de grãos de arroz em cenários de mudança climática deverá diminuir em locais de baixa latitude (tropicais) e poderá aumentar em locais de média e alta latitudes (subtropicais e temperados).

Simulando o impacto de cenários de mudança climática em arroz irrigado na China, TAO et al. (2008) e YAO et al. (2007) observaram que o decréscimo da produção de arroz, em cenários de aumento de $\mathrm{CO}_{2}$ e temperatura, variou em função da localização geográfica e com as cultivares. MATTHEWS et al. (1997) investigaram os efeitos de cenários de mudança climática em diferentes regiões da Ásia, utilizando dois modelos de simulação de

Tabela 2 - Duração da fase vegetativa, em dias, de cultivares de ciclos precoce e médio, estimada pelo método de graus-dia, considerando-se a situação atual e de cenários de aumentos na temperatura média do ar em $1{ }^{\circ} \mathrm{C}, 3^{\circ} \mathrm{C}$ e $5,8^{\circ} \mathrm{C}$, em seis localidades produtoras de arroz irrigado do Rio Grande do Sul. Adaptado de STEINMETZ et al. (2006).

\begin{tabular}{|c|c|c|c|c|c|c|c|c|c|}
\hline \multirow{3}{*}{ Local } & \multirow{3}{*}{$\begin{array}{l}\text { Data de } \\
\text { emergência }\end{array}$} & \multicolumn{8}{|c|}{ 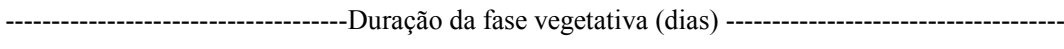 } \\
\hline & & \multicolumn{4}{|c|}{ Ciclo Precoce } & \multicolumn{4}{|c|}{ Ciclo Médio } \\
\hline & & Atual & $+1^{\circ} \mathrm{C}$ & $+3^{\circ} \mathrm{C}$ & $+5,8^{\circ} \mathrm{C}$ & Atual & $+1^{\circ} \mathrm{C}$ & $+3^{\circ} \mathrm{C}$ & $+5,8^{\circ} \mathrm{C}$ \\
\hline \multirow[t]{3}{*}{ Cachoeirinha } & $10 / 10$ & 50 & 46 & 40 & 33 & 58 & 54 & 47 & 39 \\
\hline & $10 / 11$ & 42 & 40 & 34 & 30 & 50 & 47 & 41 & 35 \\
\hline & $10 / 12$ & 38 & 36 & 32 & 28 & - & - & - & - \\
\hline \multirow[t]{3}{*}{ Pelotas } & $10 / 10$ & - & - & - & - & 70 & 60 & 50 & 44 \\
\hline & $10 / 11$ & 50 & 47 & 40 & 33 & 59 & 55 & 47 & 39 \\
\hline & $10 / 12$ & 43 & 40 & 35 & 30 & - & - & - & - \\
\hline \multirow[t]{3}{*}{ Santa Maria } & $10 / 10$ & 53 & 49 & 42 & 35 & 61 & 57 & 49 & 41 \\
\hline & $10 / 11$ & 44 & 41 & 36 & 31 & 52 & 49 & 42 & 36 \\
\hline & $10 / 12$ & 38 & 36 & 32 & 28 & - & - & - & - \\
\hline \multirow{3}{*}{$\begin{array}{l}\text { Santana do } \\
\text { Livramento }\end{array}$} & $10 / 10$ & - & - & - & - & 69 & 60 & 50 & 40 \\
\hline & $10 / 11$ & 49 & 46 & 39 & 33 & 58 & 53 & 47 & 39 \\
\hline & $10 / 12$ & 42 & 39 & 34 & 29 & - & - & - & - \\
\hline \multirow{3}{*}{$\begin{array}{l}\text { Santa Vitória do } \\
\text { Palmar }\end{array}$} & $10 / 10$ & - & - & - & - & 77 & 67 & 55 & 43 \\
\hline & $10 / 11$ & 54 & 50 & 43 & 35 & 64 & 59 & 50 & 42 \\
\hline & $10 / 12$ & 46 & 43 & 37 & 31 & - & - & - & - \\
\hline \multirow[t]{3}{*}{ Uruguaiana } & $10 / 10$ & 51 & 48 & 41 & 34 & 59 & 55 & 48 & 40 \\
\hline & $10 / 11$ & 41 & 38 & 34 & 30 & 48 & 45 & 41 & 35 \\
\hline & $10 / 12$ & 35 & 34 & 30 & 27 & - & - & - & - \\
\hline
\end{tabular}


rendimento do arroz (ORYZA1 e SIMRIW), variando a concentração de $\mathrm{CO}_{2}$ de 340 para $680 \mathrm{ppm}$, e aumentos de $+1,+2$ e $+4^{\circ} \mathrm{C}$ na temperatura do ar. Considerando apenas aumento na concentração de $\mathrm{CO}_{2}$, houve, em média, aumentos de 23,9 e 36,4\% na produtividade do arroz com os dois modelos utilizados. Quando o aumento na temperatura do ar foi incluído, houve um declínio no rendimento até uma redução de $-7,0 \% \mathrm{e}$ $-8,5 \%$, com aumento $4^{\circ} \mathrm{C}$ na temperatura. Esses valores são a média de todos os locais da Ásia e os resultados variaram de local para local. Os autores descrevem que as diferenças entre as regiões ocorreram principalmente pela diferença na quantidade de dias com temperaturas acima da ótima, dependendo do clima de cada local, resultando em maior ou menor efeito sobre a esterilidade de espiguetas e sobre a duração do ciclo de desenvolvimento das cultivares.

As altas temperaturas que ocorrem em regiões tropicais contribuem para a redução do rendimento de grãos, por diminuir o período vegetativo da cultura e por causar esterilidade de espiguetas no florescimento (PENG et al., 1995). Algumas soluções para minimizar os efeitos negativos no rendimento de arroz são sugeridas por MATTHEWS et al. (1997), como o ajuste das datas de semeadura de acordo com o local. Por exemplo, nos locais em que os verões são mais quentes, a semeadura deverá ser atrasada, a fim de evitar altas temperaturas no período do florescimento. Já em locais de altas latitudes $\left(39,9^{\circ} \mathrm{N}\right)$, onde os verões são mais amenos, o aumento na temperatura do ar tornaria possível a antecipação da data de semeadura, possibilitando dois cultivos por ano. Em um estudo similar, KRISHNAN et al. (2007) também encontraram melhores rendimentos com o atraso na data de semeadura de arroz irrigado, em locais de latitudes entre 20 e $26^{\circ} \mathrm{N}$. Outra medida de mitigação é a seleção de cultivares com maior tolerância às temperaturas elevadas, visando diminuir a esterilidade de espiguetas.

Para entender o efeito da mudança climática na esterilidade de espiguetas em arroz, os resultados de MATSUI et al. (1997) em câmaras de crescimento são de relevante interesse, pois foram considerados dois níveis de $\mathrm{CO}_{2}$ (ambiente e $+300 \mathrm{ppm}$ ) e dois níveis de temperatura (ambiente e $+4^{\circ} \mathrm{C}$ ). Os resultados mostram que, considerando somente o incremento de $\mathrm{CO}_{2}$, não houve efeito significativo nas espiguetas, pois o efeito do $\mathrm{CO}_{2}$ isoladamente é um maior número de perfilhos produtivos, tendo pouca influência na esterilidade das espiguetas. Em relação à temperatura, a antese é a fase mais sensível às temperaturas elevadas e a temperatura crítica, acima da qual a esterilidade de espiguetas começa a aumentar, ficou entre 33 e $34^{\circ} \mathrm{C}$.
Quando essa temperatura crítica é ultrapassada, mesmo por poucas horas, ocorre uma grande esterilidade de espiguetas, devido à menor deposição de grãos de pólen, e, em temperaturas extremas $\left(>40^{\circ} \mathrm{C}\right)$, ocorre esterilidade dos órgãos femininos da flor. Embora $\mathrm{o}$ efeito isolado do $\mathrm{CO}_{2}$ não tenha sido significativo, a esterilidade de espiguetas foi maior na presença de temperatura e $\mathrm{CO}_{2}$ elevados do que no caso de apenas elevada temperatura, o que sugere um efeito de aumento da temperatura da planta com o fechamento estomático no ambiente enriquecido com $\mathrm{CO}_{2}$.

\section{CONCLUSÃO}

Um aumento da concentração de $\mathrm{CO}_{2}$ atmosférico deve aumentar a produtividade de arroz. No entanto, se este aumento na concentração de $\mathrm{CO}_{2}$ vier acompanhado de aumento na temperatura do ar, como é previsto, o efeito benéfico do $\mathrm{CO}_{2}$ poderá ser anulado pelo efeito negativo das altas temperaturas. Nesse caso, as temperaturas elevadas poderão diminuir o ciclo da cultura, principalmente na fase vegetativa, aumentar a esterilidade de espiguetas e, consequentemente, reduzir a produtividade da cultura.

As limitações na produção de arroz em condições de $\mathrm{CO}_{2}$ e temperatura elevados podem ser mitigadas, pelo menos em parte, adequando a época de semeadura de acordo com as particularidades de cada local e selecionando genótipos que possuam maior resistência às altas temperaturas no florescimento.

\section{REFERÊNCIAS}

AGGARWAL, P.K.; MALL, R.K. Climate change and rice yields in diverse Agro-environments of India. II. Effect of uncertainties in scenarios and crop models on impact assessment. Climatic Change, v.52, n.3, p.331-343, 2002. Disponível em: <http://www.springerlink.com.w10050.dotlib.com.br/ content/g3h51j993m44265m/fulltext.pdf>. Acesso em: 20 ago. 2008. doi: $10.1023 / \mathrm{A}: 1013714506779$.

ANDRESEN, J.A. et al. Weather impacts on maize, soybean, and alfalfa production in the Great Lakes Region, 1895-1996. Agronomy Journal, v.93, n.5, p.1059-1070, 2001. Disponível em: <http://agron.scijournals.org/cgi/content/full/93/ 5/1059>. Acesso em: 17 nov. 2008.

ASSAD, E.D.; PINTO, H.S. Aquecimento global e a nova geografia da produção agrícola no Brasil. São Paulo: Embrapa, 2008. 84p.

IPCC [Intergovernmental Panel on Climate Change]. Climate change 2007: the physical Science Basis - Contribution of Working Group I to the Fourth assessment report of the Intergovernmental Panel on Climate Change. Cambridge: University, 2007. 996p. Disponível em: <http:// www.ipcc.ch/ipccreports/ar4-wg1.htm>. Acesso em: 22 set. 2008 . 
FLOSS, E.L. Fisiologia das plantas cultivadas: o estudo do que está por trás do que se vê. 4.ed. Passo Fundo: Universidade de Passo Fundo, 2008. 733p.

GONÇALVES, R.R.V.; ASSAD, E.D. Análise de tendências de temperatura mínima do Brasil. In: CONGRESSO BRASILEIRO DE AGROMETEOROLOGIA, 16., 2009, Belo Horizonte, MG. Anais... Belo Horizonte: SBAGRO, 2009. 1 CD-ROM.

KIMBALL, B. A. et al. Effects of increasing atmospheric $\mathrm{CO}_{2}$ on vegetation. Vegetatio, v.104-105, p.65-75, 1993. Disponível em: <http://search.nal.usda.gov/bitstream/10113/ 20068/1/IND44105061.pdf. Acesso em: 20 mar. 2009. doi: $10.1007 / \mathrm{BF} 00048145>$.

KRISHNAN, P. et al. Impact of elevated $\mathrm{CO}_{2}$ and temperature on rice yield and methods of adaptation as evaluated by crop simulation studies. Agriculture, Ecosystems and Environment, v.122, n.2, p.233-242, 2007. Disponível em: <http://www.sciencedirect.com/ science?_ob=MImg\&_imagekey=B6T3Y-4N6FNPR-1 $1 \&$ cdi $=4959 \&$ user $=687358 \&$ orig $=$ search $\&$ coverDate $=10 \%$ $2 \mathrm{~F} 31 \% 2 \mathrm{~F} 2007 \&$ sk $=998779997 \& \mathrm{view}=\mathrm{c} \& \mathrm{wchp}=\mathrm{dGLbVzz}-$ zSkzV\&md5=1782585c4d63f8cf1 1 ac3 1 ce56beb3a2\&ie $=/$ sdarticle.pdf $>$. Acesso em: 07 jul. 2008. doi:10.1016/ j.agee.2007.01.019.

LAGO, I. et al. Impact of increasing mean air temperature on the development of rice and red Rice. Pesquisa Agropecuária Brasileira, v.43, n.11, p.1441-1448, 2008. Disponível em: $<$ http://www.scielo.br/pdf/pab/v43n11/01.pdf. Acesso em: 14 ago. 2008>. doi: 10.1590/S0100-204X2008001100001.

LAL, M. et al. Vulnerability of rice and wheat yields in NW India to future changes in climate. Agricultural and Forest Meteorology, v.89, n.2, p.101-114, 1998. Disponível em: $<\mathrm{h} \mathrm{t} \mathrm{t} \mathrm{p} \mathrm{:} \mathrm{/} \mathrm{/} \mathrm{w} \mathrm{w} \mathrm{w.} \mathrm{s} \mathrm{c} \mathrm{i} \mathrm{e} \mathrm{n} \mathrm{c} \mathrm{e} \mathrm{d} \mathrm{i} \mathrm{r} \mathrm{e} \mathrm{c} \mathrm{t} \mathrm{.} \mathrm{c} \mathrm{o} \mathrm{m/}$ science? ob=MImg\&_imagekey=B6V8W-3 SX81CS-3$1 \& \_$cdi $=5881 \&$ user $=687358 \&$ orig $=$ search\&_coverDate $=02 \% 2$ F28\%2F1998\& sk=999109997 \&view $=\mathrm{c} \& w \mathrm{wh} p=\mathrm{dGLzVzzzSk}$ WA\& $\mathrm{md} 5=\mathrm{ca} 26 \mathrm{~d} 7 \mathrm{~d} 1 \mathrm{cc} 22 \mathrm{a} 38080 \mathrm{ff} 95 \mathrm{e} 1 \mathrm{~d} 4760456 \& \mathrm{ie}=/$ sdarticle.pdf $>$. Acesso em: 20 ago. 2008. doi: 10.1016/S01681923(97)00064-6.

LONG, S.P. Modification of the response of photosynthetic productivity to rising temperature by atmospheric $\mathrm{CO}_{2}$ concentrations: Has its importance been underestimated? Plant, Cell and Environment, v.14, n.8, p.729-739, 1991. Disponível em: <http://www3.interscience.wiley.com/cgi-bin/ fulltext/119351677/PDFSTART. Acesso em: 25 set. 2008. doi: 10.1111/1365-3040>.ep8115256.

LUZZARDI, R. et al. Avaliação preliminar da produtividade em campo e qualidade industrial de híbridos de arroz no Rio Grande do Sul. In: CONGRESSO BRASILEIRO DE ARROZ IRRIGADO, 4.; REUNIÃO DA CULTURA DO ARROZ IRRIGADO, 26., 2005, Santa Maria, RS. Anais... Santa Maria: SOSBAI, 2005. V.1, 567p. p.70-72.

MANN, M.E. et al. Global-scale temperature patterns and climate forcing over the past six centuries. Nature, v.392, n.6678, p.779-787, 1998. Disponível em: http:// www.nature.com/nature/journal/v392/n6678/pdf/392779a0.pdf. Acesso em: 28 ago. 2008. doi:10.1038/33859.

MANN, M.E. et al. Northern hemisphere temperatures during the past millennium: inferences, uncertainties, and limitations.
Geophysical Research Letters, v.26, n.6, p.759-762, 1999. Disponível em: http://www.ncdc.noaa.gov/paleo/pubs/millenniumcamera.pdf. Acesso em: 28 ago. 2008. doi:10.1029/ 1999GL900070.

MATSUI, T. et al. Effects of high temperature and $\mathrm{CO}_{2}$ concentration on spikelet sterility in indica rice. Field Crops Research, v.51, n.3, p.213-219, 1997. Disponível em: http:/ / w w w. s c i e n c e d i r e c t. c o m / science?_ob=MImg\&_imagekey=B6T6M-3RGTBCP-5$1 \&$ cdi $=5034 \&$ user $=687358 \&$ orig $=$ search $\&$ coverDate $=0$ 4\%2F30\%2F1997\&_sk=999489996\&view $=\mathrm{c} \& \mathrm{wchp}=\mathrm{dGLbVt}$ b-zSkWA\&md5=f6263166d88c4b16425373abe39f1bd2\&ie=/ sdarticle.pdf. Acesso em: 05 ago. 2008. doi:10.1016/S03784290(96)03451-X.

MATTHEWS, R.B. et al. Simulating the impact of climate change on rice production in Asia and evaluating options for adaptation. Agricultural Systems. v.54, n.3, p.399-425, 1997. Disponível em: <http://www.sciencedirect.com/ science? ob=MImg\& imagekey=B6T3 W-3RGSX36-S$1 \& \_$cdi $=4957 \&$ user $=687358 \&$ \& orig $=$ search \&_coverDate $=07 \%$ 2F31\%2F1997\&_sk=999459996\&view $=c \& w c h p=d G L b V t z-$ zSkzk\&md5 $=685$ e 56 bae 8 b5 db $828408172 \mathrm{ef} 19 \mathrm{dbabb} \& i \mathrm{e}=/$ sdarticle.pdf $>$. Acesso em: 20 set. 2008. doi:10.1016/S0308$521 \mathrm{X}(95) 00060-\mathrm{I}$.

PENG, S. et al. (Eds.) Climate change and rice. Los Baños: International Rice Research Institute, 1995. 377p.

ROSENBERG, N.J. et al. Microclimate: the biological environment. New York: John Wiley \& Sons, 1983. 495p.

SHEEHY, J.E. et al. (Eds). Charting new pathways to $\mathbf{C}_{4}$ rice. Los Baños: International Rice Research Institute, 2007. $422 \mathrm{p}$.

SIONIT, N. et al. Interaction of temperature and $\mathrm{CO}_{2}$ enrichment on soybean: photosynthesis and seed yield. Canadian Journal of Plant Science, v.67, n.3, p.629-636, 1987. Disponível em: $<\mathrm{ht} \mathrm{t}$ p : // a r t i c le.pu b s.n r c - c n r c . g c . c a / RPASrpv? $\mathrm{hm}=$ HInit\&calyLang $=$ eng\&journal $=$ cjps\&volume $=67 \&$ afpf $=$ cjps87-089.pdf $>$. Acesso em: 14 jun. 2008. doi:10.4141/ cjps87-089.

SIQUEIRA, O.J.W. et al. Efeitos potenciais das mudanças climáticas na agricultura brasileira e estratégias adaptativas para algumas culturas. In: LIMA, M.A. et al. Mudanças climáticas globais e a agropecuária brasileira. Jaguariúna: EMBRAPA, 2001. p.33-63.

SOLTANI, A. et al. Simulating GFDL predicted climate change impacts on rice cropping in Iran. Journal of Agricultural Science and Technology, v.3, p.81-90, 2001. Disponível em: <http://www.sid.ir/En/VEWSSID/J_pdf/84820010201.pdf>. Acesso em: 20 jan. 2009.

STEINMETZ, S. et al. Impacto do aquecimento global sobre a duração da fase vegetativa do arroz irrigado, estimada pelo método de graus-dia. In: CONGRESSO BRASILEIRO DA CADEIA PRODUTIVA DE ARROZ, 2.; REUNIÃO NACIONAL DA PESQUISA DE ARROZ, 8., 2006, Brasília. Anais... Brasília: Embrapa Arroz e Feijão, 2006. 1 CD-ROM.

STRECK, N.A. Climate change and agroecosystems: the effect of elevated atmospheric $\mathrm{CO}_{2}$ and temperature on crop growth, 
development, and yield. Ciência Rural, v.35, n.3, p.730740, 2005. Disponível em: <http://www.scielo.br/pdf/cr/v35n3/ a41v35n3.pdf>. Acesso em: 15 ago. 2008. doi: 10.1590/ S0103-84782005000300041.

STRECK, N.A. et al. Duração do ciclo de desenvolvimento de cultivares de arroz em função da emissão de folhas no colmo principal. Ciência Rural, v.36, n.4, p.1086-1093, 2006. Disponível em: <http://www.scielo.br/pdf/cr/v36n4/a07v36n4.pdf>. Acesso em: 8 maio, 2010. doi: 10.1590/S010384782006000400007 .

TAIZ, L.; ZEIGER, E. Fisiologia vegetal. 3.ed. Porto Alegre: Artemed, 2004. 719p.

TAO, F. et al. Global warming, rice production, and water use in China: developing a probabilistic assessment. Agricultural and Forest Meteorology, v.148, n.1, p.94110, 2008. Disponível em: <http://www.sciencedirect.com/ science?_ob=MImg\&_imagekey=B6V8W-4R2Y42W-1 $8 \&$ \&di $=5881 \&$ _user $=687358 \&$ orig $=$ search $\&$ _coverDate $=01$ $\% 2$ F07\%2F2008\&_sk=998519998\&view $=\mathrm{c} \& w c h p=d G L b V z b-$ zSkzk\&md5 $=558 \mathrm{a} 6122 \mathrm{e} 856 \mathrm{~d} 77 \mathrm{fc} 75 \mathrm{fe} 2 \mathrm{~b} 8 \mathrm{aeed} 80 \mathrm{f} 0 \& \mathrm{e} \mathrm{e}=/$ sdarticle.pdf $>$. Acesso em: 18 set. 2008. doi:10.1016/ j.agrformet.2007.09.012.

USDA - UNITED STATES DEPARTMENT OF AGRICULTURE, Foreign Agricultural Service. Production, supply and distribution online: custom query. USA, 2009. Disponível em: $<$ http://www.fas.usda.gov/psdonline/psdQuery.aspx. Acesso em: 20 jun. 2009>.

VU, J.C.V. et al. Effects of elevated $\mathrm{CO}_{2}$ and temperature on photosynthesis and Rubisco in rice and soybean. Plant, Cell and Environment, v.20, n.1, p.68-76, 1997. Disponível em:
$<$ http://www3.interscience.wiley.com/cgi-bin/fulltext/ 119148234/PDFSTART>. Acesso em: 03 out. 2008. doi: 10.1046/j.1365-3040.1997.d01-13.x.

YANG, L. et al. Seasonal changes in the effects of free-air $\mathrm{CO}_{2}$ enrichment (FACE) on dry matter production and distribution of rice (Oryza sativa L.). Field Crops Research, v.98, n.1, p.12-19, 2006. Disponível em: <http://www.sciencedirect.com/ science?_ob=MImg\&_imagekey=B6T6M-4JD0H3M-1$9 \&$ \& cdi $=5034 \&$ \&user $=687358 \&$ orig $=$ browse\&_coverDate $=07 \%$ 2F01\%2F2006\&_sk=999019998\&view $=\mathrm{c} \& \mathrm{w} \mathrm{chp}=\mathrm{dGLzVtz}-$ zSkWb\&md5=94 d8b6004012640340c2f29e7a208d4d\&ie=/ sdarticle.pdf>. Acesso em: 12 nov. 2008. doi:10.1016/ j.fcr.2005.11.003.

YAO, F. et al. Assessing the impacts of climate change on rice yields in the main rice areas of China. Climatic Change, v.80, n.3, p.395-409, 2007. Disponível em: <http:// www.springerlink.com.w 10050 .dotlib.com.br/content/ yv7p342336427572/fulltext.pdf $>$. Acesso em: 23 out. 2008. doi: $10.1007 / \mathrm{s} 10584-006-9122-6$.

WIEGAND, C.L.; CUELLAR, J.A. Duration of grain filling and kernel weight of wheat as affected by temperature. Crop Science, v.21, n.1, p.95-101, 1981.

ZISKA, L.H.; McCLUNG, A. Differential response of cultivated and weedy (red) rice to recent and projected increases in atmospheric carbon dioxide. Agronomy Journal, v.100, n.5, p.1259-1263, 2008. Disponível em: <http://link.periodicos.capes.gov.br/ sfxlc13?url_ver=Z39.88-2004\&url_ctx_fmt=infofi/ fmt:kev:mtx:ctx\&ctx enc =info: of $\bar{i} /$ enc:UTF$8 \& \mathrm{ctx}_{\text {_ ver }}=\mathrm{Z} 39.882004 \& \mathrm{rfr}$ id $=\mathrm{info}: \mathrm{sid} /$ sfxit.com:azlist\&sfx.ignore date threshold $=1 \&$ rft.object id $=9549$ $25375849 \&$ rft.object_portfolio_id $=\& s v c . f u l l t e x t=y e s>$. Acesso em: 19 maio, 2009. doi: 10.2134/agronj2007.0324. 